\title{
An Automatic System for Enumeration of Colony-Forming Units by the Surface Plate Method
}

\author{
Yoshimi Benno, ${ }^{1}$ Shigenari Kunisaki ${ }^{2}$ and Tomotari Mrtsuoka ${ }^{2,3}$ \\ 1 Japan Collection of Microorganisms, and ${ }^{2}$ Frontier Research Program, \\ RIKEN, Wako, Saitama, 351-01, \\ ${ }^{3}$ Department of Biomedical Science, Faculty of Agriculture, \\ The University of Tokyo, Bunkyo-ku, Tokyo, 113 \\ (Received for publication, June 14, 1989)
}

\begin{abstract}
A device for mechanical dilution and application of specimens and counting microbial colonies was developed. The device consists of a transport apparatus which transports petri dishes from one side of the apparatus to the other in such a way that the plate and lid of petri dishes are entirely separated at a particular stage. A diluting and inoculating system performs 10 -fold dilutions of specimens from $10^{-1}$ to $10^{-8}$ dilutions and inoculation of the diluents. The inoculum is applied to the agar surface of preselected petri plates by an application system. Both a diluting and inoculating system and an application system can be connected to the apparatus. In this way a maximum of 25 agar plates can be handled. This new automatic system for enumeration of colony-forming units by the surface plate method is more accurate and reliable than the customary conventional methods.
\end{abstract}

Key words : automatic system; colony forming unit

Many types of non-selective and selective agar in petri plates, are used for the bacterial analysis and determination of colony-forming units (CFU) of clinical specimens $(6)$, microflora $(2,10)$ and food and water $(4)$. When large numbers of agar plates have to be used for these bacterial analyses, the handling of petri dishes and diluting of samples are difficult to perform manually and mistakes might occur. Furthermore, application of the inoculum was very difficult to perform accurately because the agar plates were often divided into 3 or 4 parts (9). There is little information concerning automation of bacterial examinations by the surface plate method. We therefore developed a system in which handling of petri dishes, dilution of samples, and application of inocula are mechanized.

The automatic system consists of 3 main parts: i) a transport apparatus, ii) a diluting and inoculating system, and iii) an application system. The latter 2 parts can be connected individually to the transport apparatus. 


\section{MATERIALS AND METHODS}

Transport apparatus. Figure 1 shows the transport apparatus, diluting and inoculating system, and the application system. This machine was designed in such a way that the plate and lid of petri dishes $10 \mathrm{~cm}$ in diameter are entirely separated when arriving at the delivery station. The transport apparatus is shown schematically in Fig. 2. Inoculated plates covered with lids are placed manually in the right stacking tower (I). When the starter button is pushed, the plate of the lowest petri dish in the right stacking tower drops onto the plate guide rail (IV) with a guide (III) fastened to the electric-driving chain and is pushed by the guide onto the delivery table $(\mathrm{V})$. The lid is pushed by the hopper to the lid guide rail (II). The lid guide rail is located behind the diluting and inoculating system and the application system. Thus, the plate is entirely free of the lid in this position. The guide now touches an electric switch in the transport apparatus, which stops the motor for an adjustable period of time, as well as a switch which pushes the delivery table and stacking table (VII). In the upper position, the plates on the delivery table touches inoculating the system in turn. After an adjustable period of time, the motor starts again and is released by a guide. The next guide pushes the plate of the second petri dish. At the same time, the lid of the second petri dish is pushed onto the lid guide rail. After the plate has been pushed by the plate guide rail onto the stacking table, the plate is again matched with the lid brought by the lid guide rail. The first petri dish is stacked by three one-way moving, self-locking pins connected to the shafts of the left stacking tower (VI). These pins prevent the dish from falling off the stacking table. One movement takes approximately 10 min per plate.

Diluting and inoculating system. Details of the diluting and inoculating system are indicated in Fig. 3. The system consists of eight stainless pipettes and eight tubes with an anaerobic diluent. Each pipette also has a thin stainless tube through which $\mathrm{CO}_{2}$ can flow. The diluents can be mixed by the flow of $\mathrm{CO}_{2}$. The specimens are diluted from $10^{-1}$ to $10^{-8}$ by this system. Firstly, 5 to $6 \mathrm{ml}$ of $10^{-1}$ diluent is sucked up by a stainless pipette. One $\mathrm{ml}$ of the $10^{-1}$ diluent in the stainless pipette is inoculated into the next tube with $9 \mathrm{ml}$ of the diluent. The other dilutions are also performed by the same procedure. A drop $(0.05 \mathrm{ml})$ of the diluent can be applied to the plate. The distance between the tip of the stainless pipette and the surface of the agar plate on the delivery table in its upper position is $4-5 \mathrm{~mm}$, depending on the thickness of the agar layer. If this distance is smaller, the drops of samples might not fall from the tip of the stainless pipettes onto the inoculated agar surface. If the distance is greater, the drops might roll on the agar surface, which can lead to bacterial contamination. The stainless pipettes are repeatedly (2 or 3 times) washed by $70 \%$ ethanol and sterile water and are sterilized by the special heater for $10 \mathrm{~min}$ (Fig. 4A).

Application system. The application system consists of two applicators with three bent stainless rods and an applicator with four rods (Fig. 5A). The bent sterile stainless rods are activated by a switch connected to the plate guide rail, which guides 


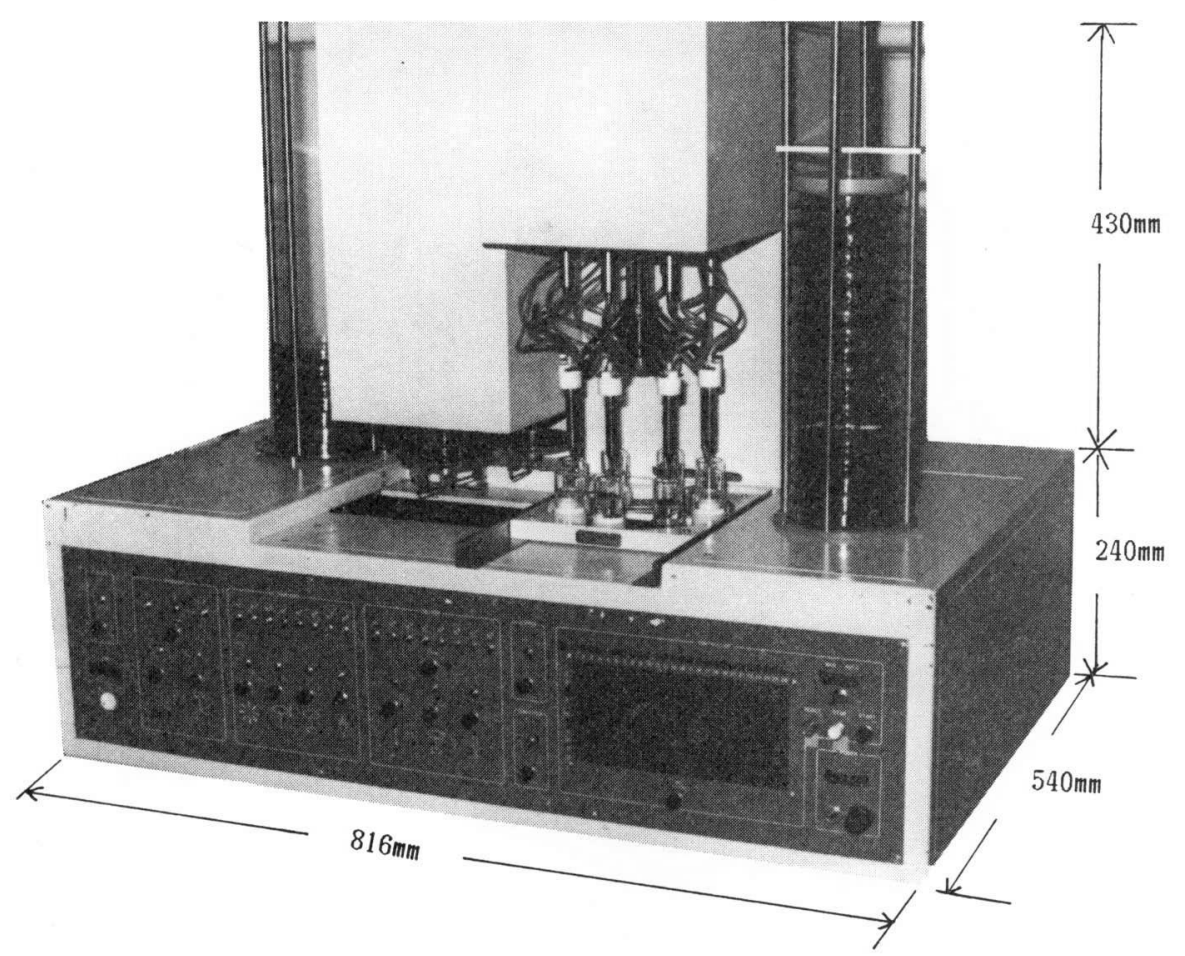

Fig. 1. Transport apparatus, diluting and inoculating system, and the application system.

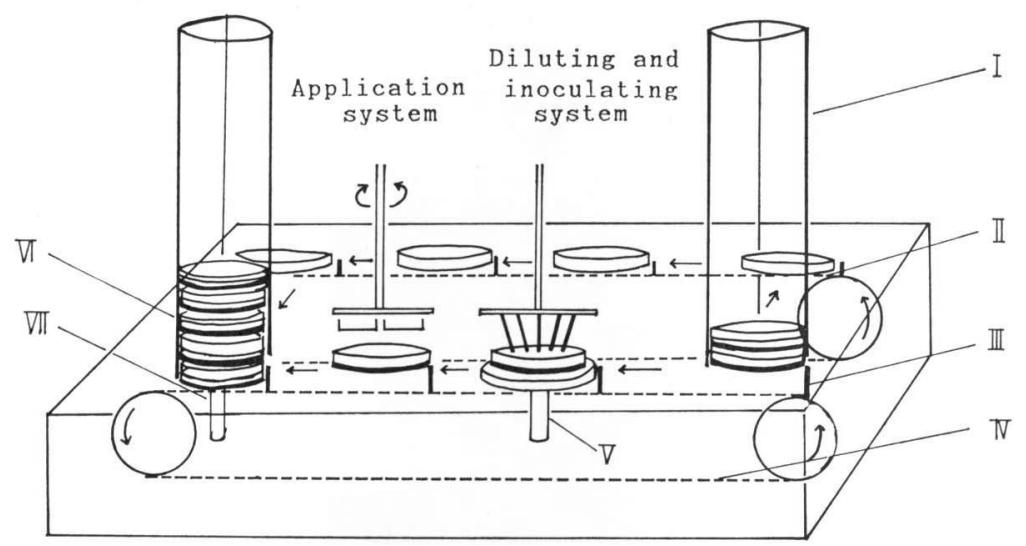

Fig. 2. Schematic diagram of the transport apparatus. Stacking tower: 25 petri dishes (consisting of plate and lid) for inoculation are placed manually into this stacking tower (I); lid guiding rail (II); guider fastened on an electromotor-driving chain (III); plate guiding rail (IV); delivery table with petri dish lifter (V). Stacking tower with one-way moving self-locking pins; 25 petri dishes stacked mechanically have to be taken out manually (VI); stacking table (VII). 


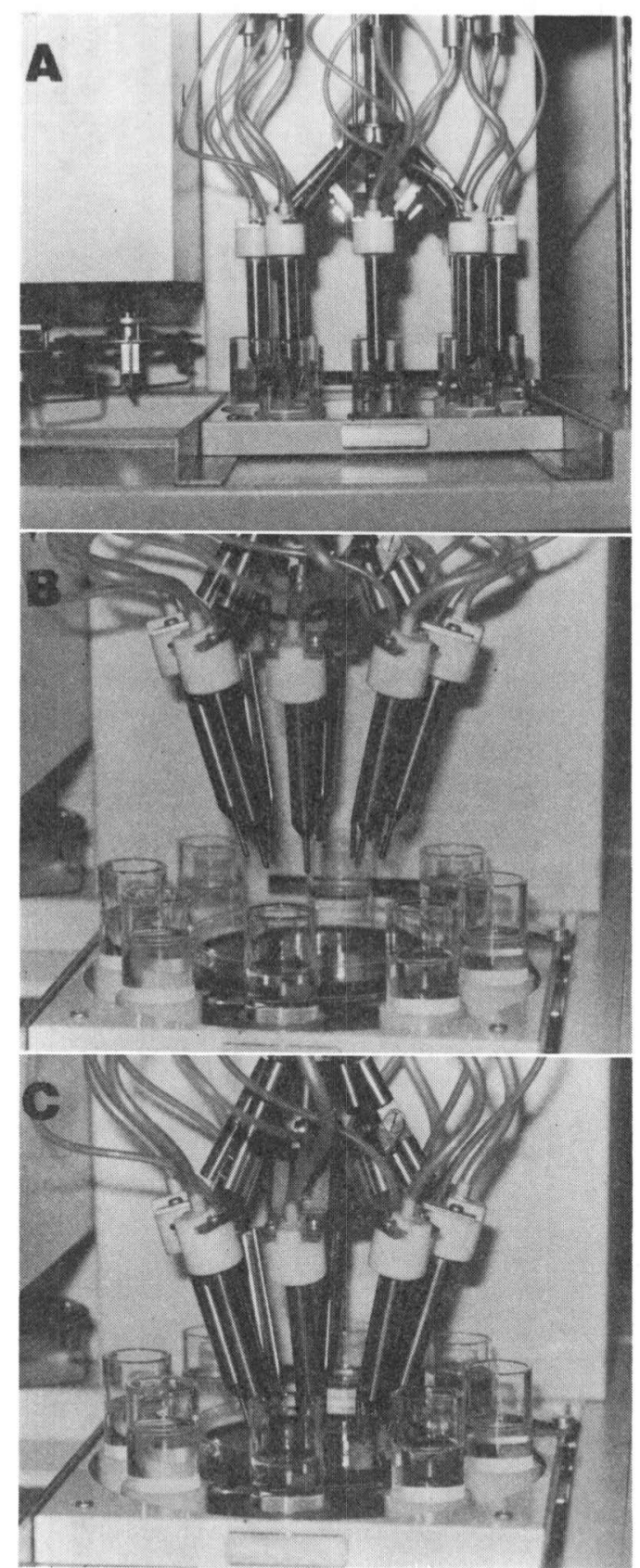

Fig. 3. Diluting and inoculating system. Diluting (A), selecting the inoculation tips (B), and inoculation on the surface of agar (C). 


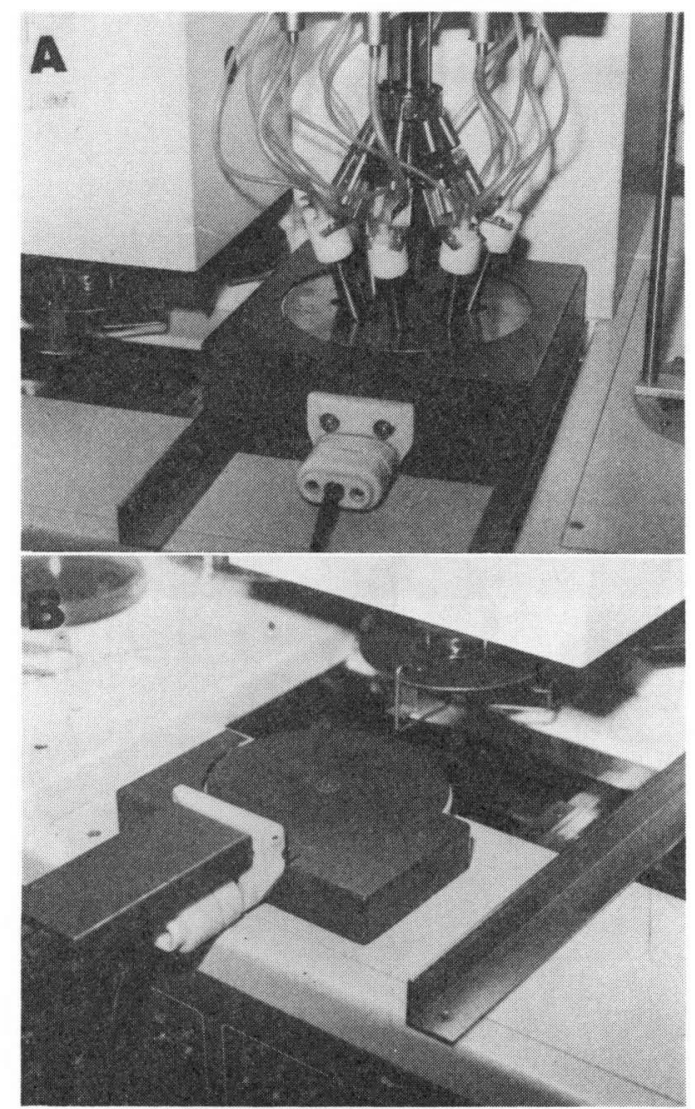

Fig. 4. Sterile system. Special heater for the stainless pipettes (A) and special heater for the bent stainless rods (B).

the plate to be inoculated. When the plate to be inoculated with three diluents $\left(10^{-6}, 10^{-7}\right.$, and $\left.10^{-8}\right)$ stops in the position, an applicator with three sterile rods pushes down (Fig. 5B). Movement of the rods is performed three times at angles of $60^{\circ}, 90^{\circ}$, and $120^{\circ}$, continuously (Fig. 5C). Application of four diluents to the plates is also performed at each angle of $30^{\circ}, 60^{\circ}$, and $90^{\circ}$, continuously. The system is connected to the transport apparatus in the same way as the diluting and inoculating system. The movements of this system take 5-6 sec per plate. The bent stainless rods are sterilized by a special heating-plate (Fig. 4B).

Procedure of the automatic bacterial counting system. The frame with the stainless pipettes is connected to the transport apparatus. Twenty-five inoculated agar plates are placed in the right stacking tower. The diluting and inoculating system is started automatically by pressing a special button until each pipette delivers the sample suspension. Transport of the plates by the transport apparatus and appli- 

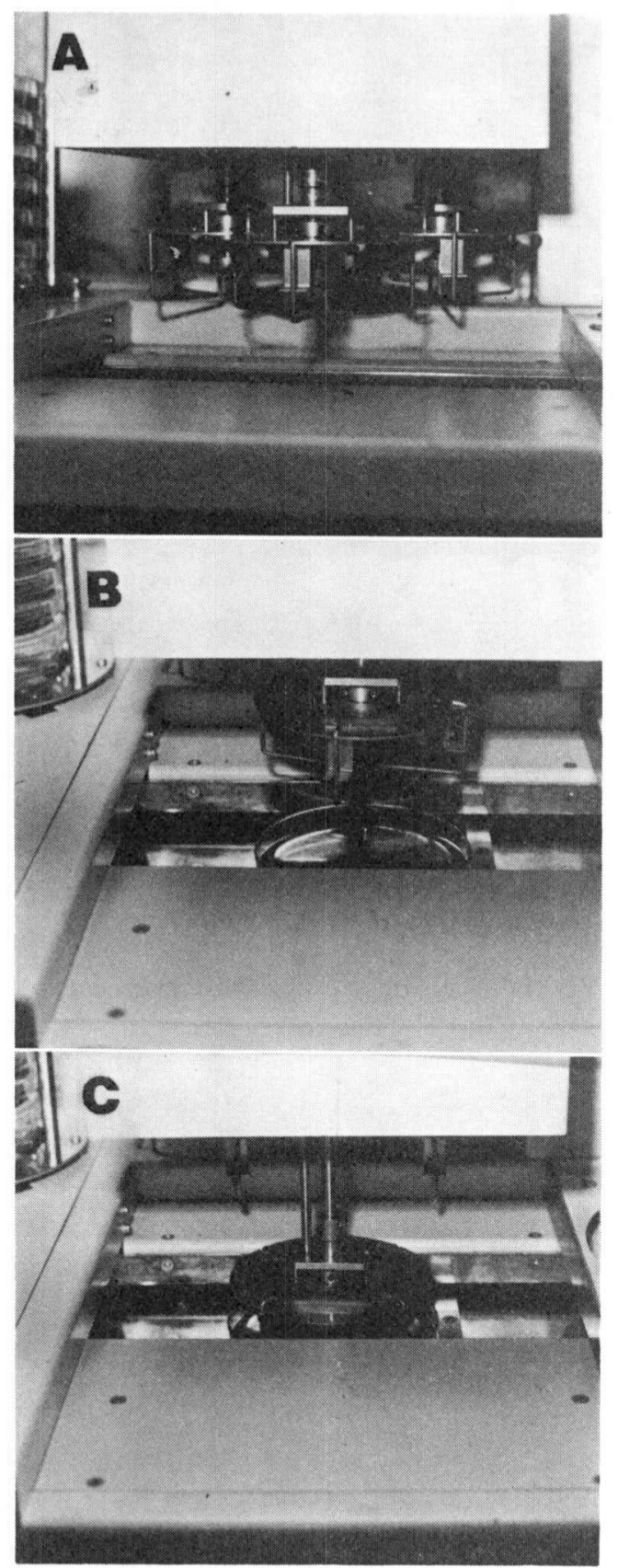

Fig. 5. Application system. Applicators with bent sterile rods (A); an applicator pushes down on the surface of agar (B); movement of the rods on the surface of agar (C). 
cation of samples takes 7-8 sec per plate. The operator feeds plates into the right stacking tower and moves the diluent-inoculated plates from the left stacking tower to the incubator.

Six strains of Bifidobacterium longum, Bacteroides vulgatus, Clostridium perfringens, Lactobacillus acidophilus, Escherichia coli, and Enterococcus faecalis were used for comparing the manual method with this automated system to evaluate the feasibility of the latter. One colony of each strain on an Eggerth-Gagnon (EG) agar plate (10) was suspended in EG-liver-Fildes solution (3) filled with $100 \% \mathrm{CO}_{2}$. After incubation at $37^{\circ} \mathrm{C}$ for $12 \mathrm{hr}$, the optical density of all cultures was adjusted to that of a McFarland No. 5 nephelometric standard. Except for B. longum and B. vulgatus, the suspensions were further diluted by dilution $\mathrm{A}(10)$ and adjusted to $10^{6}$ cells per $\mathrm{ml}$. The following media (10) were used for bacterial counting: EG agar; glucoseblood-liver (BL) agar; Trypticase soy (BBL) agar with 5\% horse blood as the nonselective media and bifidobacteria-selective (BS) agar; bacteroides-selective (NBGT) agar; neomycin-Neglar (NN) agar; lactobacilli-selective (LBS) agar; DHL (Eiken) agar for Enterobacteriaceae- and streptococci-selective (TATAC) agar as selective media. For the spread plate counts, triplicate plates were used for each strain. EG agar, BL agar, BS agar, NBGT agar, NN agar, and LBS agar were incubated at $37^{\circ} \mathrm{C}$ for three days in an anaerobic steel-wool jar (11) filled with $100 \% \mathrm{CO}_{2}$.

After incubation, the number of colonies of each strain was counted on all media. The bacterial count per $\mathrm{ml}$ was calculated from the sum of the counts of each strain. The data was expressed as mean $\pm \mathrm{SD}$ of bacterial counts per ml from counts of triplicate plates.

\section{RESULTS AND DISCUSSION}

The evaluation of microbial enumeration using the surface plate method has

Table 1. Comparison of numbers of each species detected by the automated system and the manual system

\begin{tabular}{|c|c|c|c|}
\hline Microorganism tested & Agar plate & Automated system & Manual system \\
\hline & EG & $3.8 \pm 0.5$ & $3.4 \pm 0.3$ \\
\hline \multirow[t]{2}{*}{ Bacteroides vulgatus $a)$} & $\mathrm{BL}$ & $3.2 \pm 0.4$ & $3.4 \pm 0.2$ \\
\hline & NBGT & $2.8 \pm 0.4$ & $3.0 \pm 0.3$ \\
\hline Bifidobacterium & EG & $1.6 \pm 0.4$ & $1.4 \pm 0.2$ \\
\hline \multirow[t]{2}{*}{ adolescentis $\left.{ }^{a}\right)$} & $\mathrm{BL}$ & $2.1 \pm 0.3$ & $2.3 \pm 0.3$ \\
\hline & $\mathrm{BS}$ & $1.3 \pm 0.3$ & $1.5 \pm 0.2$ \\
\hline Clostridium & $\mathrm{EG}$ & $4.1 \pm 0.3$ & $3.9 \pm 0.2$ \\
\hline \multirow[t]{2}{*}{ perfringens ${ }^{b)}$} & $\mathrm{BL}$ & $4.0 \pm 0.2$ & $4.1 \pm 0.2$ \\
\hline & $\mathrm{NN}$ & $4.0 \pm 0.5$ & $3.8 \pm 0.3$ \\
\hline Lactobacillus & $\mathrm{BL}$ & $1.6 \pm 0.4$ & $1.7 \pm 0.3$ \\
\hline acidophilus ${ }^{b)}$ & LBS & $1.6 \pm 0.3$ & $1.8 \pm 0.2$ \\
\hline \multirow[t]{2}{*}{ Escherichia colib) } & $\mathrm{TS}$ & $2.3 \pm 0.4$ & $2.4 \pm 0.2$ \\
\hline & $\mathrm{DHL}$ & $1.9 \pm 0.2$ & $1.8 \pm 0.2$ \\
\hline Enterococcus & $\mathrm{TS}$ & $2.3 \pm 0.3$ & $2.3 \pm 0.2$ \\
\hline faecalis $^{b)}$ & TATAC & $2.1 \pm 0.2$ & $2.0 \pm 0.2$ \\
\hline
\end{tabular}

a) $10^{8} \times$ mean bacterial counts per $\mathrm{ml}$ of broth \pm SD grown on three same agar plates.

b) $10^{6} \times$ mean bacterial counts per $\mathrm{ml}$ of broth $\pm \mathrm{SD}$ grown on three same agar plates. 
been carried out in various fields $(1,5,7,8,12,13)$. The automatic system described herein provides a convenient way to analyze the intestinal microflora in humans and animals. The comparison of bacterial numbers in each medium by both methods is given in Table 1. The numbers detected by the automated system were similar to those by the manual system. In this way a maximum of 25 agar plates can be handled. We also consider that this new system should be improved to shorten the treatment time for diluting of sample or sterilizing the tips of inoculator or the bent stainless rods. This new automatic system for colony-formingunit enumeration by the surface plate method is more accurate and reliable than the manual system. The results in this study indicate that the new system is highly promising for use in bacterial counting. Further studies on the usefullness of this system are under way.

\section{REFERENCES}

(1) Clark, D.S. 1971. Studies on the surface plate method of counting bacteria. Can. J. Microbiol. 17: 943-946.

(2) Clark, R.T.J. 1977. Methods for studying gut microbes, p. 1-33. In R.T.J. Clarke and T. Bauchop (eds.), Microbial ecology of the gut, Academic Press, New York.

(3) Fildes, P. 1920. New medium for the growth of B. influenza. Br. J. Exp. Pathol. 1: 129-130.

(4) Harrigan, W.F., and M.E. McCance. 1976. Laboratory methods in food and dairy microbiology, Academic Press, New York.

(5) Harris, R.F., and L.E. Sommers. 1968. Plate-dilution frequency technique for assay of microbial ecology. Appl. Microbiol. 16: 330-334.

(6) Isenberg, H.D., J.A. Washington II, A. Balows, and A.C. Sonnenwirth. 1985. Collection, handling, and processing of specimens, p. 73-98. In E.H. Lennette, A. Balows, W.J. Hausler Jr., and H.J. Shadowy (eds.), Manual of clinical microbiology, 4th ed., American Society for Microbiology, Washington, D.C.

(7) Kennedy, J.E., Jr., P.E. Phillips, and J.L. Oblinger. 1980. Effect of stored pre-poured plates on microbial enumeration using the surface plate method. J. Food Prot. 43: 592-594.

(8) Kramer, J.M., and R.J. Gilbert. 1978. Enumeration of micro-organisms in food: a comparative study of five methods. J. Hyg. 81 : 151-159.

(9) Mitsuoka, T., K. Ohno, Y. Benno, K. Suzuki, and K. Namba. 1976. Die Faekalflora bei Menshen. V. Mitteilung: Vorgleich des neu entwickelten Verfahrens mit dem bisherigen üblichen Verfahren zur Darmfloraanlyse. Zentralbl. Bakteriol. Hyg. I Abt Orig. A234: 219-233.

(10) Mistuoka, T., T. Sega, and S. Yamamoto. 1965. Eine verbesserte Methodik der qualitativen und quantitativen Analyse der Darmflora von Menschen und Tieren. Zentralbl. Bakteriol. Parasitenkd. Infektionskr. Hyg. I Abt Orig. 195: 455-469.

(11) Parker, C.A. 1955. Anaerobiosis with iron wool. Aust. J. Exp. Biol. Med. Sci. 33: $33-37$.

(12) Tan, A.-T., R.B. Maxcy, and W.W. Stroup. 1983. Colony-forming unit enumeration by a plate-mpn method. J. Food Prot. 46: 836-841.

(13) Thomas, Y.O., W.J. Lulves, and A.A. Kraft. 1981. A convenient surface plate method for bacteriological examination of poultry. J. Food Sci. 46: 1951-1952. 